\title{
Normative data of the SF-12 health survey with validation using postmyocardial infarction patients in the Dutch population
}

\author{
Floortje Mols $\cdot$ Aline J. Pelle $\cdot$ Nina Kupper
}

Accepted: 2 February 2009/Published online: 26 February 2009

(C) The Author(s) 2009. This article is published with open access at Springerlink.com

\begin{abstract}
Objective To gain Dutch population norms for the Short Form-12 (SF-12), a generic health status questionnaire, in a random sample of the general population and to validate these in postmyocardial infarction (MI) patients.

Methods 2,301 respondents from the general population and 459 post-MI patients completed the Short Form-36 (SF-36), which was used to calculate SF-12 scores.

Results The SF-12 summary scores correlated highly with SF-36 summary scores, demonstrating that these scores explain the same amount of variance in health status. Significant sex differences $(P<.001)$ existed for both the physical component summary (PCS) and the mental component summary (MCS). Multivariate analysis of variance showed a main effect of age in oblique (PCS-12: $P<.001$; MCS-12: $P<.001)$ and orthogonally rotated PCS scores (PCS-12_uc: $P<.001$; MCS-12_uc: $P=.07$ ). As expected, post-MI patients reported statistically significant and clinically relevant poorer mental $(P<.001)$ and physical functioning $(P<.001)$. Differences were less pronounced for MCS and PCS derived from orthogonal rotation data. When controlling for covariates, MI did not significantly affect PCS-12_uc anymore in orthogonally rotated data, while PCS-12_uc was affected by fewer covariates compared with PCS- 12 .

Conclusions This study presents Dutch population norms for the SF-12 in a large random population sample obtained from both oblique and orthogonal PCA rotation methods,
\end{abstract}

F. Mols $(\square) \cdot$ A. J. Pelle $\cdot$ N. Kupper

CoRPS-Center of Research on Psychology in Somatic diseases,

Department of Medical Psychology, Tilburg University,

Warandelaan 2, PO Box 90153, 5000 LE Tilburg,

The Netherlands

e-mail: F.Mols@uvt.nl revealing systematic differences between the results based on these two methods. Furthermore, this study demonstrates the discriminative validity of the SF- 12 by showing that post-MI patients differ significantly from the normative population on PCS-12 scores.

Keywords SF-12 - Normative data .

Myocardial infarction $\cdot$ Health status $\cdot$ Rotation method

\begin{tabular}{|c|c|}
\hline \multicolumn{2}{|c|}{ Abbreviations } \\
\hline MI & Myocardial infarction \\
\hline PCS & Physical component summary \\
\hline MCS & Mental component summary \\
\hline SF-36 & Short Form-36 \\
\hline SF-12 & Short Form-12 \\
\hline COPD & Chronic obstructive pulmonary disease \\
\hline BMI & Body mass index \\
\hline $\mathrm{CHF}$ & Chronic heart failure \\
\hline PAD & Peripheral arterial disease \\
\hline PCI & Percutaneous coronary intervention \\
\hline CABG & Coronary artery bypass graft \\
\hline PCA & Principle components analysis \\
\hline
\end{tabular}

\section{Introduction}

Health status is an important outcome in medical care, both from the patient's and health care provider's perspective, and is often measured with the Short Form-36 (SF-36) questionnaire [1-4]. However, an important concern in psychological assessment of clinical populations is to minimize response burden of patients, stimulating the pursuit of developing shorter questionnaires. A shorter and thus more user-friendly alternative to the SF-36 is the Short 
Form-12 (SF-12) [5]. The SF-12 measures physical and mental health by means of two summary scores; a physical component summary (PCS) and mental component summary (MCS) [6].

The SF-12 can be employed in multiple ways, i.e., SF-12 is often used to compare health status between two groups of patients, to identify predictors of health status, and to determine health status in a specific disease population. The predicament with both the SF-36 and SF-12 component summary scores is that they are somewhat difficult to interpret, because of the weighting of items to calculate PCS and MCS [5, 6]. Normative data could facilitate the interpretation of results, because these can be used to determine whether groups or individuals score above or below average for their nationality, age or sex [7]. While SF-36 normative data, according to age and sex, are available for the Dutch population [8], normative data for the SF-12 are not. Therefore, the goal of this study was to gain nationality-specific population norms for the SF-12, according to age and sex, in a large random sample of the Dutch population. In order to further validate the SF-12 (discriminative validity) and demonstrate the relevance and possible application of these Dutch norm scores, we compared health status of postmyocardial infarction (MI) patients with our newly calculated Dutch normative scores.

\section{Methods}

Setting and participants

The sample comprised a random selection of 2,301 adults from the general Dutch population residing in the Southern provinces of The Netherlands (population of approximately 4 million). Quota sampling was applied to ensure that different age and sex groups were equally represented in the sample. This meant that equal numbers of men and women in different age groups were sampled (e.g., $N$ of men aged 30-39 years $=N$ of women aged 60-69 years). Research assistants were responsible for distributing the questionnaires and were instructed to collect an equal number of questionnaires from each age and sex subcohort, without further specification of educational or income level. Participants were approached personally or by phone. After explaining the purpose of the study, participants received an informed consent form and a questionnaire, which were returned to the research assistants in closed envelopes. The questionnaires were entered into the database by others, guaranteeing anonymity. Returned questionnaires did not contain any explicit identifiers (i.e., names) but rather, were coded by number for purposes of data collection tracking. Approval for this study was obtained from a local ethics committee (protocol number 2006/1101).

Additionally, data was used from a prospective follow-up study in patients recovering from MI. Patients hospitalized for acute MI ( $n=459)$ were recruited between May 2003 and May 2006 from four teaching hospitals (Catharina Hospital, Eindhoven; St. Elisabeth Hospital, Tilburg; TweeSteden Hospital, Tilburg; and St. Anna Hospital, Geldrop) in the Southern provinces of The Netherlands. Inclusion criteria were age $>30$ years and hospitalization due to acute MI. Criteria for diagnosis of MI were troponin I levels more than twice the upper limit, with typical ischemic symptoms (e.g., chest pain) lasting for more than $10 \mathrm{~min}$ or electrocardiogram (ECG) evidence of ST segment elevation or new pathological Q-waves. For patients without typical angina, the day of MI onset was identified as the day during hospitalization with peak troponin I levels $>1.0$ and ECG evidence of ST segment elevation or new pathological Qwaves. Exclusion criteria were significant cognitive impairments (e.g., dementia) and severe medical comorbidities that increased the likelihood of early death, such as malignant cancer. All participants were approached to participate on a voluntary basis, and could withdraw from the study at any moment without implications for future treatment. The study protocol was approved by the institutional review boards of the participating hospitals (protocol number M03/1302). Written consent was obtained from all study participants.

\section{Measures}

\section{Sociodemographic and clinical characteristics} in a random sample of the Dutch population

Demographic variables included age, sex, marital status, and classified educational level. Clinical variables were obtained from the questionnaires as well using purposedesigned questions, and included smoking, height, weight, and illnesses for which participants had received an official diagnosis, including hypertension, diabetes mellitus, renal disease, chronic obstructive pulmonary disease (COPD), hyperlipidemia, and cardiovascular disease.

\section{Sociodemographic and clinical characteristics in post-MI patients}

Demographic variables included age, sex, marital status, and educational level. Clinical variables associated with post-MI prognosis were obtained from the patients' medical records. These included cardiac treatment [prior percutaneous coronary intervention (PCI) or coronary artery bypass graft (CABG)], body mass index (BMI), hyperlipidemia, diabetes mellitus, renal insufficiency, 
COPD, chronic heart failure (CHF), peripheral arterial disease (PAD), history of hypertension, and current smoking status (self-report).

\section{Clinical significant depression and anxiety}

People are, in general, very capable in determining the presence of depression, as a single question (e.g., "Are you depressed?") was found to predict the presence of major depressive disorder very well [9]. Likewise, we obtained information on clinically diagnosed anxiety and depression in the general Dutch population by the following question in our questionnaire; "Did a doctor/medical specialist diagnose you with one of the following conditions?" This was followed by a list that included both anxiety disorder as well as depression.

In post-MI patients, the World Health Organizationauthorized Dutch version of the Composite International Diagnostic Interview (CIDI) [10, 11], a fully structured diagnostic interview, was used 3 months post-MI, to determine lifetime diagnoses of major depressive disorder and anxiety disorder (consisting of panic disorder, social phobia, and/or generalized anxiety disorder). These disorders were assessed using the definitions and criteria of Diagnostic and Statistical Manual of Mental Disorders (DSM-IV) [12]. The CIDI has acceptable interrater and test-retest reliability for most nonpsychotic diagnoses, including major depressive disorder [13, 14].

\section{Health status}

In order to provide a shorter alternative to the SF-36, the SF-12 health survey was developed in 1994 [15] in the USA, and was purposely designed for large-scale measurements for which the SF-36 was too lengthy. The SF-12 contains 12 items derived from the SF-36, including one or two items from each of the eight SF-36 subscales (physical functioning, role limitations due to physical health, bodily pain, general health perceptions, vitality, social functioning, role limitations due to emotional problems, and mental health) [8]. These 12 items are used to construct the physical component summary (PCS) and the mental component summary (MCS) [15].

In this study, health status was assessed by version 1 of the Dutch SF-36 questionnaire (in both the norm and post-MI population), which was used to calculate SF-12 component summary scores [5]. Internal consistency reliability coefficients have been reported for each SF-36 subscale, ranging from 0.62 to 0.96 with a median of 0.80 [16]. Test-retest reliability of the SF-36 ranges from 0.43 to 0.90 with a median of 0.64 after 6 months in the general population [16]. In post-MI patients, health status was assessed 3 months post-MI by means of the SF-36.

\section{Statistical analysis}

Calculating SF-36 domain scores

Following the SF-36 scoring instructions, items were reversed or recalibrated when necessary [17]. Then, the raw domain scores were calculated, also including those respondents who had missing values on no more than half of the domain's score items. In a third step, the raw domain scores were transformed to a $0-100$ scale. For those respondents who had missing values on more than half of the domain's score items, no data substitution algorithms were used to handle missing data. For the eight dimensions of the SF-36, $0.5 \%(n=12)$ of cases were missing for physical functioning, $0.7 \%(n=12)$ of cases were missing for social functioning, $1.8 \%(n=42)$ of cases were missing for role limitations due to physical health, $2.1 \%$ $(n=48)$ of cases were missing for role limitations due to emotional problems, $0.9 \%(n=20)$ of cases were missing for mental health, $0.8 \%$ (19) of cases were missing for vitality, $0.7 \%(n=17)$ of cases were missing for bodily pain, and $1.2 \%$ (27) of cases were missing for general health perception.

\section{Calculating PCS-36 and MCS-36 component summary scores}

PCS and MCS scores are essentially summations of the weighted domain scores. To obtain PCS and MCS scores, a dedicated procedure was used, described in the SF-36 summary scales manual [18]. The domain scores were first standardized, and then principle components analysis (PCA) was employed to obtain the domain weights needed to construct PCS and MCS. More specifically, the eight domains were factor-analyzed twice. Once using the standardized approach, orthogonal rotation (varimax), which by definition assumes factors to be uncorrelated, and once using an approach, oblique rotation (promax), which by definition allows factors to be correlated. The SF-36 summary measures manual [18] and various papers show that, when using the orthogonal method, the mental and physical component summaries demonstrate low to very low empirical correlations $[19,20]$. Papers that have used the oblique rotation method have reported high correlations between the mental and physical component summary scores [15, 21-23]. The reason for employing orthogonal rotation, as stated in the SF-36 manual, was a more straightforward interpretation of each component, driven by the goal of testing the construct validity of the SF-36. The use of oblique rotation in this and previous papers on the other hand had different reasons, in that it was based on theoretical arguments about the a priori knowledge of the relation between mental and physical health. We decided 
therefore to analyze the data twice because of the apparent discrepancy between the manual guidelines advocating orthogonal rotation for their purposes, and the observations from the literature that mental and physical health in fact are related [15, 21-23]. The domain weights (resulting from orthogonal and oblique rotation of the eight SF-36 subscales) are presented in Table 1, where a comparison is made between the new Dutch weights and the goldstandard US weights (adapted from [24]). Note that the US weights were obtained from PCA using orthogonal rotation (varimax). The observable differences in Table 1 are largely due to the difference in rotation. When we applied an orthogonal rotation, despite factors being correlated, our Dutch weights resembled US weights closely. Additionally, the negative loadings largely disappear in case of oblique rotation, removing the negative effect of better mental health on physical health and vice versa, as Farivar et al. suggested [25].

The final step was to calculate raw PCS-36 and MCS-36 component scores by summing up all weighted domain scores according to the weights in the two-factor solution based on orthogonal and oblique rotation. Summary scores were then transformed so that they would have standard deviation of 10 (multiply by 10) and mean of 50 (add 50).

\section{Calculating PCS-12 and MCS-12 component summary} scores

In accordance with the developers' recommendations [6] we selected the appropriate SF-12 items from the SF-36 to reproduce the PCS-12 and MCS-12 scores in the general Dutch population and a large sample of Dutch post-MI patients. Instructions were followed to reverse item scores so that a higher score always represented better health status. Next, indicator variables were made for each answer option, and PCS-12 and MCS-12 indicator variables weights were calculated by regressing the indicator variables against, respectively, the PCS-36 and MCS-36 component summary scores. Table 2 shows the indicator variable weights for this Dutch normative population. These weights should be used to calculate SF-12 component summary scores when comparing data with this normative population. Again, we calculated the weights for each rotation method. When comparing the obtained weights with the weights published by Farivar et al. based on US data, the magnitude of the weights is comparable [25].

Rotation-specific PCS-12 and MCS-12 component summary scores were finally computed by summing all weighted indicator variable scores, and standardizing them by adding the constants (specific for PCS-12 and MCS-12, respectively) from the regression analyses described above. For the validation sample of Dutch post-MI patients we used our normative population weights and constants in the calculation of PCS-12 and MCS-12 scores. When presenting our results, the extension "_uc" (which stands for uncorrelated) will indicate that summary scores were derived from orthogonal rotation PCA.

Statistical analyses

Correspondence of SF-12 scores with the SF-36 scores was checked by examining the proportion of the variance in PCS-36 and MCS-36 component summary scores that was explained by the 12 items of the SF-12 $\left(R^{2}\right)$. In addition, we examined the (Spearman's) correlations between the summary component scores of the SF-36 and SF-12, and between the summary component scores within the SF-12. In addition, we correlated (Spearman's) SF-12 summary

Table 1 Factor score coefficients from the SF-36 resulting from PCA using two rotation methods

\begin{tabular}{|c|c|c|c|c|c|c|}
\hline & \multicolumn{3}{|l|}{ PCS-36 } & \multicolumn{3}{|l|}{ MCS-36 } \\
\hline & \multicolumn{2}{|c|}{ The Netherlands } & \multirow{2}{*}{$\begin{array}{l}\text { USA } \\
\text { Orthogonal }\end{array}$} & \multicolumn{2}{|c|}{ The Netherlands } & \multirow{2}{*}{$\begin{array}{l}\text { USA } \\
\text { Orthogona }\end{array}$} \\
\hline & Oblique & Orthogonal & & Oblique & Orthogonal & \\
\hline Physical functioning & 0.328 & 0.411 & 0.424 & -0.042 & -0.216 & -0.230 \\
\hline Role physical & 0.244 & 0.258 & 0.351 & 0.079 & -0.023 & -0.123 \\
\hline Bodily pain & 0.312 & 0.369 & 0.318 & -0.081 & -0.169 & -0.097 \\
\hline General health perception & 0.276 & 0.291 & 0.250 & 0.028 & -0.073 & -0.016 \\
\hline Energy/vitality & 0.095 & 0.000 & 0.029 & 0.265 & 0.283 & 0.235 \\
\hline Social functioning & 0.113 & 0.044 & -0.008 & 0.238 & 0.229 & 0.269 \\
\hline Role emotional & -0.054 & -0.193 & -0.192 & 0.341 & 0.431 & 0.434 \\
\hline Mental health & -0.069 & -0.220 & -0.221 & 0.384 & 0.490 & 0.486 \\
\hline
\end{tabular}

Note: US weights are the result of PCA with orthogonal rotation and those from the US SF-36 developers (adapted from [17, 24]). Dutch factor score coefficients were obtained from two PCA analyses, one employing orthogonal rotation (standardized method) and one using oblique rotation 
Table 2 Regression weights for calculating SF-12 PCS and MCS scores
Note: The PCS and MCS component scores are calculated by first adding all weighted answer categories, and then standardizing this score by adding the regression constant (oblique: PCS

constant $=61.14074, \mathrm{MCS}$

constant $=61.63188$;

orthogonal: PCS

constant $=58.752, \mathrm{MCS}$ constant $=59.289$ ). For each question, indicator variables were constructed based on answer categories (see

"Methods" section). Each row represents the answer categories per subscale. For example "PFQ2_A1" stands for answer option 1 on question 2 of the physical functioning subscale

\begin{tabular}{|c|c|c|c|c|}
\hline \multirow[t]{2}{*}{ SF-12 answer category } & \multicolumn{2}{|c|}{ Oblique rotation method } & \multicolumn{2}{|c|}{ Orthogonal rotation method } \\
\hline & PCS-12 & MCS-12 & PCS-12_uc & MCS-12_uc \\
\hline \multicolumn{5}{|l|}{ Physical functioning } \\
\hline PFQ2_A1 & -4.719 & 0.879 & -6.148 & 3.072 \\
\hline PFQ2_A2 & -2.352 & 0.306 & -2.987 & 1.334 \\
\hline PFQ4_A1 & -6.461 & 0.459 & -7.757 & 3.016 \\
\hline PFQ4_A2 & -2.755 & 0.142 & -3.346 & 1.338 \\
\hline \multicolumn{5}{|l|}{ Physical role } \\
\hline rpQ2_A1 & -2.858 & -0.863 & -3.041 & 0.070 \\
\hline rpQ3_A1 & -3.911 & -1.035 & -4.246 & 0.407 \\
\hline \multicolumn{5}{|l|}{ Emotional role } \\
\hline REQ2_A1 & 0.594 & -5.599 & 3.016 & -6.910 \\
\hline REQ3_A1 & 0.760 & -6.043 & 3.336 & -7.438 \\
\hline \multicolumn{5}{|l|}{ Bodily pain } \\
\hline BPQ2_A1 & -12.066 & 0.371 & -14.346 & 4.565 \\
\hline BPQ2_A2 & -10.585 & 1.787 & -12.952 & 5.433 \\
\hline BPQ2_A3 & -7.174 & 1.432 & -8.835 & 3.850 \\
\hline BPQ2_A4 & -3.998 & 0.558 & -4.926 & 2.083 \\
\hline \multicolumn{5}{|l|}{ General health } \\
\hline GHQ1_A1 & -9.956 & -2.736 & -10.904 & 0.756 \\
\hline GHQ1_A2 & -6.679 & -0.868 & -7.642 & 1.556 \\
\hline GHQ1_A3 & -3.447 & -0.318 & -3.982 & 0.928 \\
\hline GHQ1_A4 & -1.665 & -0.126 & -1.958 & 0.508 \\
\hline \multicolumn{5}{|l|}{ Vitality } \\
\hline VTQ2_A1 & -1.895 & -6.232 & 0.307 & -6.640 \\
\hline VTQ2_A2 & -2.652 & -6.333 & -0.657 & -6.441 \\
\hline VTQ2_A3 & -1.907 & -4.611 & -0.420 & -4.775 \\
\hline VTQ2_A4 & -1.238 & -3.303 & -0.168 & -3.407 \\
\hline VTQ2_A5 & -0.755 & -1.645 & -0.208 & -1.705 \\
\hline \multicolumn{5}{|l|}{ Mental health } \\
\hline MHQ3_A1 & 0.341 & -7.603 & 2.966 & -8.817 \\
\hline MHQ3_A2 & 0.711 & -9.250 & 4.149 & -10.865 \\
\hline MHQ3_A3 & 0.189 & -7.191 & 2.801 & -8.272 \\
\hline MHQ3_A4 & 0.075 & -4.234 & 1.598 & -4.858 \\
\hline MHQ3_A5 & -0.085 & -2.119 & 0.676 & -2.439 \\
\hline MHQ4_A1 & 2.695 & -11.164 & 6.535 & -13.668 \\
\hline MHQ4_A2 & 0.525 & -11.018 & 4.526 & -12.763 \\
\hline MHQ4_A3 & 0.007 & -8.347 & 2.932 & -9.513 \\
\hline MHQ4_A4 & 0.492 & -4.847 & 2.231 & -5.650 \\
\hline MHQ4_A5 & 0.119 & -2.325 & 0.920 & -2.632 \\
\hline \multicolumn{5}{|l|}{ Social functioning } \\
\hline SFQ2_A1 & -4.560 & -7.505 & -1.885 & -7.110 \\
\hline SFQ2_A2 & -3.387 & -8.255 & -0.464 & -8.331 \\
\hline SFQ2_A3 & -2.866 & -4.661 & -1.368 & -4.328 \\
\hline SFQ2_A4 & -1.316 & -2.348 & -0.495 & -2.346 \\
\hline
\end{tabular}

component scores (calculated once for each rotation method) with demographic characteristics, i.e., age, sex, educational level, and social situation.
Data on patient and disease characteristics were compared between the Dutch normative population and the post-MI patient population using independent samples 
$t$-tests for continuous variables and chi-squared analyses for categorical variables.

Mean SF-12 component summary scores were calculated for the Dutch normative population, separately for each rotation method (Tables 3 and 4). Norm scores were categorized by sex (total group; male; female) and by age (total group; 30-39 years; 40-49 years; 50-59 years; 60-69 years; 70-79 years). Sex and age differences were formally tested using the nonparametric Mann-Whitney $U$-test (sex), and a multivariate analysis of variance (MANOVA) with a Dunnett's T3 post hoc analysis for unequal variances (categorized age).

To evaluate the discriminative properties of the Dutch SF-12, MANOVA was used to examine the differences in SF-12 component summary scores between the normative and post-MI patient samples. Analyses were performed for the total group and for each age category separately, for both variants of the summary scores. To evaluate clinical relevance of the observed differences in health status scores, effect sizes were calculated using Cohen's $d$ [26, 27]. Cohen's $d$ represents the differences between means (i.e., normative sample versus post-MI patients) divided by the pooled standard deviation. An effect size ranging from 0.00 to 0.20 is considered negligible to small, from 0.20 to 0.50 small to moderate, from 0.50 to 0.80 large, and $>0.80$ very large [27]. In a next step, demographics and clinical and psychological risk factors (see Table 5 for an overview of these variables) were added as covariates to examine the extent to which the differences between the normative population and the post-MI population might be explained by these covariates. For this purpose, we dichotomized educational level into low (high school and below) versus higher education. All statistical analyses were performed using SPSS version 14.0. A $P$-value $<.05$ was used for all tests to indicate statistical significance.

\section{Results}

The sample from the general Dutch population comprised 2,301 middle-aged adults ( $49.1 \%$ men; age: mean 55.2 years, SD 14.3 years). Descriptives are presented in Table 5.

Table 3 Dutch mean age- and sex-standardized scores for the mental and physical component summary of the SF-12 (oblique rotation method)

\begin{tabular}{|c|c|c|c|c|c|c|}
\hline \multirow[t]{2}{*}{ Age (years) } & \multicolumn{2}{|c|}{ Total group $(n=2,013)$} & \multicolumn{2}{|c|}{ Women $(n=1,019)$} & \multicolumn{2}{|c|}{$\operatorname{Men}(n=992)$} \\
\hline & MCS-12 & PCS-12 & MCS-12 & PCS-12 & MCS-12 & PCS-12 \\
\hline 30-39 & $n=395$ & $n=395$ & $n=198$ & $n=198$ & $n=197$ & $n=197$ \\
\hline Mean score & 51.08 & 53.77 & 49.80 & 52.76 & 52.36 & 54.78 \\
\hline SD & 9.22 & 6.44 & 9.61 & 6.71 & 8.65 & 6.02 \\
\hline $95 \% \mathrm{CI}$ & $50.17-51.99$ & $53.13-54.4$ & $48.46-51.15$ & $51.82-53.70$ & $51.14-53.57$ & $53.93-55.63$ \\
\hline $40-49$ & $n=421$ & $n=421$ & $n=222$ & $n=222$ & $n=198$ & $n=198$ \\
\hline Mean score & 51.07 & 53.17 & 49.79 & 51.97 & 52.57 & 54.55 \\
\hline SD & 9.09 & 6.99 & 9.58 & 7.48 & 8.25 & 6.13 \\
\hline $95 \% \mathrm{CI}$ & $50.20-51.94$ & $52.50-53.84$ & $48.52-51.06$ & $50.98-52.96$ & $51.41-53.73$ & $53.69-55.41$ \\
\hline $50-59$ & $n=436$ & $n=436$ & $n=216$ & $n=216$ & $n=220$ & $n=220$ \\
\hline Mean score & 50.89 & 50.71 & 49.48 & 49.58 & 52.27 & 51.83 \\
\hline SD & 8.85 & 9.07 & 9.41 & 9.66 & 8.04 & 8.32 \\
\hline $95 \% \mathrm{CI}$ & $50.06-51.72$ & $49.86-51.57$ & $48.22-50.74$ & $48.28-50.87$ & $51.20-53.34$ & $50.72-52.93$ \\
\hline $60-69$ & $n=391$ & $n=391$ & $n=194$ & $n=194$ & $n=197$ & $n=197$ \\
\hline Mean score & 51.30 & 50.86 & 50.07 & 49.76 & 52.50 & 51.95 \\
\hline SD & 8.76 & 8.26 & 8.84 & 8.76 & 8.53 & 7.60 \\
\hline $95 \% \mathrm{CI}$ & $50.43-52.17$ & $50.04-51.68$ & $48.82-51.32$ & $48.52-51.00$ & $51.31-53.70$ & $50.88-53.02$ \\
\hline 70-79 & $n=370$ & $n=370$ & $n=189$ & $n=189$ & $n=180$ & $n=180$ \\
\hline Mean score & 47.71 & 44.22 & 46.59 & 42.16 & 48.87 & 46.46 \\
\hline SD & 10.52 & 11.69 & 10.43 & 11.96 & 10.55 & 10.99 \\
\hline $95 \% \mathrm{CI}$ & $46.63-48.78$ & $43.02-45.41$ & $45.09-48.09$ & $40.44-43.88$ & $47.32-50.42$ & $44.84-48.07$ \\
\hline Total group & $n=2,013$ & $n=2,013$ & $n=1,019$ & $n=1,019$ & $n=992$ & $n=992$ \\
\hline Mean score & 50.45 & 50.66 & 49.19 & 49.38 & 51.78 & 52.00 \\
\hline SD & 9.36 & 9.23 & 9.64 & 9.74 & 8.89 & 8.46 \\
\hline $95 \% \mathrm{CI}$ & $50.04-50.87$ & $50.26-51.06$ & $48.59-49.78$ & $48.78-49.98$ & $51.22-52.33$ & $51.48-52.53$ \\
\hline
\end{tabular}

Abbreviations $\mathrm{SD}=$ standard deviation, $\mathrm{CI}=$ confidence interval, $\mathrm{PCS}=$ physical component summary, MCS = mental component summary 
Table 4 Dutch mean age- and sex-standardized scores for the mental and physical component summary of the SF-12 (orthogonal rotation method)

\begin{tabular}{|c|c|c|c|c|c|c|}
\hline \multirow[t]{2}{*}{ Age (years) } & \multicolumn{2}{|c|}{ Total group $(n=2,013)$} & \multicolumn{2}{|c|}{ Women $(n=1,019)$} & \multicolumn{2}{|c|}{$\operatorname{Men}(n=992)$} \\
\hline & MCS-12_uc & PCS-12_uc & MCS-12_uc & PCS-12_uc & MCS-12_uc & PCS-12_uc \\
\hline 30-39 & $n=395$ & $n=395$ & $n=198$ & $n=198$ & $n=197$ & $n=197$ \\
\hline Mean score & 49.78 & 54.06 & 48.67 & 53.37 & 50.89 & 54.76 \\
\hline SD & 9.70 & 6.53 & 10.31 & 7.09 & 8.92 & 5.85 \\
\hline $95 \%$ CI & $48.82-50.74$ & $53.42-54.71$ & $47.22-50.11$ & $52.38-54.37$ & $49.63-52.14$ & $53.93-55.58$ \\
\hline $40-49$ & $n=421$ & $n=421$ & $n=222$ & $n=222$ & $n=198$ & $n=198$ \\
\hline Mean score & 49.99 & 53.37 & 48.95 & 52.45 & 51.22 & 54.41 \\
\hline SD & 9.18 & 6.68 & 9.78 & 7.31 & 8.30 & 5.77 \\
\hline $95 \%$ CI & $49.11-50.87$ & $52.73-54.01$ & $47.66-50.24$ & $51.48-53.41$ & $50.05-52.38$ & $53.60-55.22$ \\
\hline $50-59$ & $n=436$ & $n=436$ & $n=216$ & $n=216$ & $n=220$ & $n=220$ \\
\hline Mean score & 50.72 & 50.51 & 49.51 & 49.71 & 51.91 & 51.31 \\
\hline SD & 9.04 & 9.45 & 9.48 & 9.91 & 8.45 & 8.91 \\
\hline $95 \% \mathrm{CI}$ & $49.87-51.57$ & $49.63-51.40$ & $48.24-50.78$ & $48.28-50.87$ & $50.78-53.03$ & $50.12-52.49$ \\
\hline 60-69 & $n=391$ & $n=391$ & $n=194$ & $n=194$ & $n=197$ & $n=197$ \\
\hline Mean score & 51.13 & 50.54 & 50.13 & 49.70 & 52.12 & 51.37 \\
\hline SD & 8.73 & 8.24 & 9.02 & 9.06 & 8.35 & 7.28 \\
\hline $95 \%$ CI & $50.26-52.00$ & $49.72-51.36$ & $48.85-51.40$ & $48.42-50.98$ & $50.95-53.29$ & $50.35-52.39$ \\
\hline 70-79 & $n=370$ & $n=370$ & $n=189$ & $n=189$ & $n=180$ & $n=180$ \\
\hline Mean score & 49.50 & 44.06 & 48.99 & 42.06 & 50.01 & 46.26 \\
\hline SD & 8.49 & 11.15 & 9.50 & 11.63 & 9.48 & 10.17 \\
\hline $95 \% \mathrm{CI}$ & $46.53-50.47$ & $42.93-45.20$ & $47.62-50.35$ & $40.39-43.73$ & $48.61-51.40$ & $44.76-47.76$ \\
\hline Total group & $n=2,013$ & $n=2,013$ & $n=1,019$ & $n=1,019$ & $n=992$ & $n=992$ \\
\hline Mean score & 50.24 & 50.63 & 49.25 & 49.60 & 51.26 & 51.71 \\
\hline SD & 9.24 & 9.21 & 9.63 & 9.88 & 8.71 & 8.30 \\
\hline $95 \%$ CI & $49.83-50.64$ & $50.22-51.03$ & $48.65-49.84$ & $48.99-50.20$ & $50.72-51.81$ & $51.19-52.23$ \\
\hline
\end{tabular}

Abbreviations $\mathrm{SD}=$ standard deviation, $\mathrm{CI}=$ confidence interval, $\mathrm{PCS}=$ physical component summary, $\mathrm{MCS}=$ mental component summary

Confirmation of the relation between SF-12 and SF-36

As expected, results showed that there was almost complete overlap between the SF-12 and SF-36, as the proportion of the variance $\left(R^{2}\right)$ in PCS-36 and MCS-36 explained by SF-12 items was, respectively, $94 \%$ and $93 \%$ in case of oblique rotation, and $92 \%$ for both component summaries in case of orthogonal rotation. Furthermore, we found that, in case of oblique rotation, PCS-36 and PCS-12 correlated .93 and MCS-36 and MCS-12 summary scores correlated .96. Using orthogonal rotation, correlations were .90 and .94 for the relation between PCS-36 and PCS-12, and MCS-36 and MCS-12, respectively. When allowing a correlation between PCS-12 and MCS-12 (by PCA with oblique rotation), the SF-12 derived summary scores PCS12 and MCS-12 showed substantial correlation $(r=.61)$, but this was no different from the SF-36 summary scores $(r=.59)$. Nonparametric correlations (Spearman's rho) between MCS-12 and PCS-12 and demographic variables sex, age, education level, and marital status for both rotation methods are presented in Table 6. Sex and age differences were assessed for all component summary scores, calculated using oblique and orthogonal rotation method. The Mann-Whitney test showed the presence of significant sex differences for PCS-12 $(P<.001)$ and PCS12 uc $(P<.001)$ and MCS-12 $(P<.001)$ and MCS-12_uc $(P<.001)$. MANOVA showed a significant main effect of age (PCS-12: $F=73.270, \quad P<.001 ; \quad$ MCS-12: $F=$ $10.067, P<.001)$. Post hoc analysis showed that, for PCS12 , subjects in age groups 30-39 years and 40-49 years were similar to each other $(P=.90)$, and different from the other age groups $(\mathrm{ps}<.001)$. Furthermore, PCS-12 scores for age groups 50-59 years and 60-69 years were similar $(P=1.00)$, and the oldest participants (aged 70-79 years) differed from all other groups $(\mathrm{ps}<.001)$. The mental component summary scores (MCS-12) were of equal magnitude for the first four age groups, while the oldest participants (70-79 years) scored significantly lower than all other groups $(P<.001)$. For PCS-12_uc similar age differences were found $(F=81.308, P<.001)$ and post hoc analysis revealed the same pattern of similarities and differences between age groups as the norm scores based 
Table 5 Baseline characteristics for the Dutch normative population and post-MI patients

\begin{tabular}{|c|c|c|c|}
\hline Characteristic & $\begin{array}{l}\text { Normative } \\
\text { population } \\
(n=2,301)\end{array}$ & $\begin{array}{l}\text { Post-MI } \\
\text { patients } \\
(n=459)\end{array}$ & $P$-value \\
\hline \multicolumn{4}{|l|}{ Demographics } \\
\hline $\begin{array}{l}\text { Age, years, } \\
\text { mean (SD) }\end{array}$ & $55.2(14.3)$ & $59.5(11.2)$ & $<.001$ \\
\hline Male sex & $49.1(1,128)$ & $80.0(367)$ & $<.001$ \\
\hline No partner & $16.5(380)$ & $17.6(81)$ & .55 \\
\hline Low education & $30.2(694)$ & $45.3(208)$ & $<.001$ \\
\hline \multicolumn{4}{|l|}{ Cardiovascular history } \\
\hline $\begin{array}{l}\text { Cardiovascular } \\
\text { disease }^{\#}\end{array}$ & $12.8(289)$ & $100(459)$ & $<.001$ \\
\hline \multicolumn{4}{|l|}{ Clinical factors } \\
\hline Diabetes mellitus & $5.6(126)$ & $14.2(65)$ & $<.001$ \\
\hline Hypercholesterolemia & $1.0(22)$ & 34.7 (157) & $<.001$ \\
\hline Hypertension & $9.3(208)$ & 38.7 (176) & $<.001$ \\
\hline COPD & $4.8(110)$ & $7.8(36)$ & $<.01$ \\
\hline Renal failure & $0.4(9)$ & $3.5(16)$ & $<.001$ \\
\hline Current smoking & $20(459)$ & $38.0(174)$ & $<.001$ \\
\hline $\begin{array}{l}\text { BMI, kg/m², } \\
\quad \text { mean }(\mathrm{SD})\end{array}$ & $25.6(3.9)$ & $26.9(4.0)$ & $<.001$ \\
\hline \multicolumn{4}{|c|}{ Psychological risk factors } \\
\hline Anxiety disorder & $1.8(40)$ & $7.1(29)$ & $<.001$ \\
\hline Depressive disorder & $4.0(89)$ & $17.1(70)$ & $<.001$ \\
\hline
\end{tabular}

Results are presented as \% (n), unless otherwise stated

\# Cardiovascular disease includes previous MI, angina pectoris, atherosclerosis, chronic heart failure, peripheral arterial disease or any procedure relating to the heart (coronary artery bypass graft or percutaneous coronary intervention)

$\mathrm{BMI}=$ body mass index; $\mathrm{COPD}=$ chronic obstructive pulmonary disease

Table 6 Nonparametric correlations between MCS-12 and PCS-12 and demographic characteristics

\begin{tabular}{lccllc}
\hline & \multicolumn{2}{l}{ Oblique rotation } & & \multicolumn{2}{l}{ Orthogonal rotation } \\
\cline { 2 - 3 } \cline { 6 - 6 } \cline { 5 - 6 } & PCS-12 & MCS-12 & & PCS-12_uc & MCS-12_uc \\
\hline Sex & -0.15 & -0.16 & & -0.10 & -0.12 \\
Categorized age & -0.29 & -0.09 & & -0.33 & 0.01 (ns) \\
Education* & -0.19 & -0.09 & & -0.18 & -0.03 \\
Marital status* & -0.13 & -0.13 & & -0.10 & -0.09 \\
\hline
\end{tabular}

All correlations were significant at a $P<.01$ level, except age $\times$ MCS12_uc. *These variables are dichotomized, i.e., education: lower versus higher education, and marital status: having a partner versus having no partner

on the oblique rotation method. However, for MCS-12_uc no age differences were found $(F=2.130, P=.08)$, with post hoc analysis showing no group differences (ps $>.13$ ).
Population norms for the Dutch population differed from those of Dutch post-MI patients

The sample of Dutch post-MI patients comprised 459 individuals $(80 \%$ men, age: mean 59.5 years, SD 11.2 years) (Table 5). Compared with the normative Dutch sample, post-MI patients were older, more often male, and had a lower educational level (all ps $<.001$ ). Not surprisingly, comorbid diseases and biomedical risk factors were more prevalent in the post-MI population (all ps $<.01$ ), as well as having a history of anxiety disorder or depressive disorder, which was more common in post-MI patients (ps $<.001$ ). None of the post-MI patients had been diagnosed with $\mathrm{CHF}$ or PAD. Five percent of post-MI patients underwent a CABG or PCI.

Post-MI patients had statistically significant poorer scores than the general population on MCS-12 (means 50.6 ( \pm 9.3$)$ versus $44.6( \pm 12.4) ; F=105.445, P<.001)$ and PCS-12 (means 50.7 $( \pm 9.2)$ versus $43.5( \pm 10.7) ; \quad F=$ 168.222, $P<.001$ ) of the SF-12 (Fig. 1). These differences were also clinically relevant, with Cohen's $d$ being large for MCS-12 and PCS-12 (0.55 and 0.72, respectively) [27]. Orthogonal rotation derived summary scores essentially resulted in the same differences. However, differences in MCS-12_uc (means $50.2( \pm 9.2)$ versus 46.2 ( \pm 12.0$)$; $F=53.044, P<.001)$ and PCS-12_uc (means $50.6( \pm 9.2)$ versus $44.6( \pm 10.1) ; F=127.456, P<.001)$ between the post-MI patients and normative control group were less pronounced, with Cohen's $d$ being 0.37 for MCS-12_uc (i.e., small to moderate) and 0.62 (i.e., large) for PCS-12_uc. When stratifying data by age categories, results demonstrated that in all age categories post-MI patients reported a lower mental (all ps <.01) and physical (all ps <.001) health status (oblique rotation), except for the oldest subgroup (aged 70-79 years) in which MCS-12 $(F=1.974$, $P=.16)$ and PCS-12 $(F=1.814, P=.18)$ scores were equal to the normative age group. Orthogonal rotation derived summary scores showed slightly different results for MCS-12_uc, as there were no differences in mental health status between MI patients and normative controls in the youngest age cohort $(F=1.994, P=.16)$ or in the oldest age group $(F=1.241, P=.27)$. PCS-12_uc was significantly impaired in all MI patients (ps <.001) except for those in the oldest age group $(F=1.320, P=.25)$.

Adding the covariates to the nonstratified multivariable model revealed that all covariates except hyperlipidemia $(P=.14)$ and lower educational level $(P=.25)$ significantly affected the physical component summary score (all ps $<.01)$. The mental component summary score significantly covaried with all variables (all ps $<.05$ ) except for BMI $(P=.43)$, hyperlipidemia $(P=.20)$, and lower educational level $(P=.25)$. After controlling for all potential confounders, MI patients still differed 

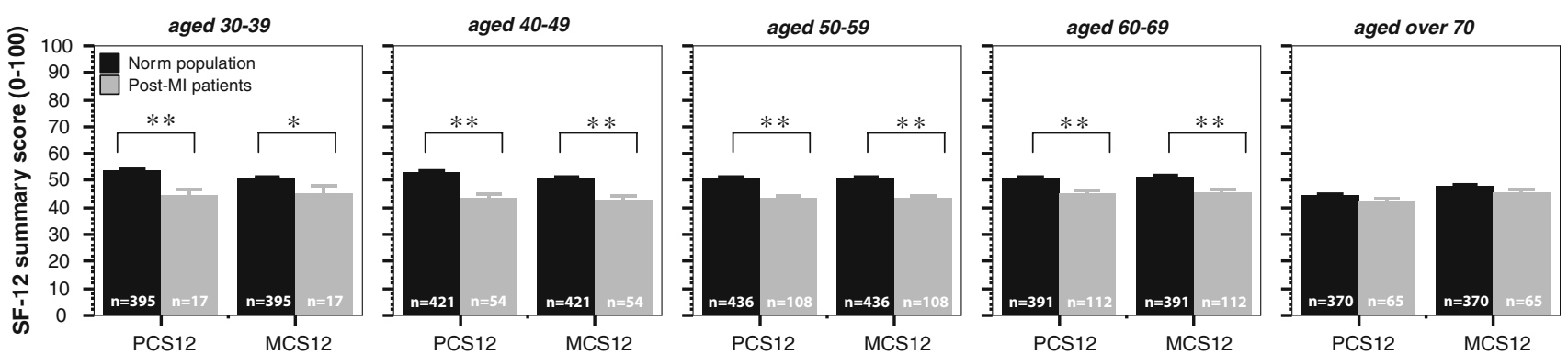

Fig. 1 Comparison of SF-12 data (oblique rotation) from the general Dutch population with a sample of post-MI patients. Black bars represent the normative population; gray bars represent the post-MI

population. Error bars: 1 SEM. *Significant at $P<.01$ level, **Significant at $P<.001$ level

significantly from the norm population on PCS-12 scores $(P=.04)$, while differences in MCS-12 scores were no longer significant $(P=.16)$. For the orthogonal rotation derived summary scores, quite a few differences were observed compared with the oblique rotation derived summary scores. In addition to hyperlipidemia $(P=.21)$ and lower educational level $(P=.37)$, PCS-12_uc was also not affected by the covariates anxiety $(P=.55)$ and depression $(P=.99)$. The other variables significantly covaried with PCS-12_uc (all ps <.03). MCS-12_uc significantly covaried with all variables (all ps $<.05)$ except for BMI $(P=.20)$, hypertension $(P=.06)$, hyperlipidemia $(P=.34)$, and lower educational level $(P=.33)$. After controlling for all potential confounders, data based on PCA with orthogonal rotation revealed that MI patients did no longer differ significantly from the norm population on PCS-12_uc scores $(P=.07)$ or MCS-12_uc scores $(P=.37)$.

\section{Discussion}

This study confirmed the close resemblance of the SF-36 and SF-12 in the Dutch population. In line with previous studies [23], significant sex and age differences existed for both PCS-12 and MCS-12. For PCS-12, this was valid for both rotation methods. For MCS-12, norm scores (both orthogonal and oblique rotated data) did not show an agerelated decline (MCS-12 scores decreased on average 3 points, but this decrease was found completely in the oldest age group), whereas this might have been expected based on a previous study in Greeks in which there was a steady decrease of in total 6 points on MCS-12 between ages 18 years and $65+$ years [23]. Post-MI patients had statistically significant and clinically relevant poorer scores than the general Dutch population on MCS-12 and PCS-12. After controlling for all covariates, MI patients still differed significantly from the normative population on PCS12 scores (oblique rotation only), while MCS-12 scores were comparable (both rotation methods).

The findings of the present study regarding the oblique rotation results concur with a study conducted in the UK in which PCS-36 and MCS-36 scores were also found to be highly correlated to the PCS-12 and MCS-12, respectively [21]. Several other studies confirmed these findings across different European countries [15, 22, 23]. The current study also showed that, within the SF-12, the MCS and PCS summary scores were substantially correlated, and this was also found for the SF-36 summary scores. Correlations between SF-12 PCS and MCS scores in the IQOLA study, an SF-12 validation study in nine European countries including The Netherlands, were quite small, which was due to the use of US PCS-36 and MCS-36, which were constructed based on PCA with orthogonal rotation, precluding a higher correlation between factors [15]. Furthermore, correlations between the two SF-12 summary scores and correlations between the SF-36 summary scores were also close to zero in a Chinese population, which is to be expected as they followed guidelines to employ orthogonal rotation [19]. Conversely, a recent study by Spindler and colleagues in a Danish sample of cardiac patients, reported a significant correlation of .33 between PCS-36 and MCS-36, while using US weights to calculate the summary scores [28]. This correlation seems intrinsic to the PCS and MCS scores, as all items, both physical and mental, contribute to both component scores.

In this study we assumed that responses to the SF-12 items embedded within the SF-36 are the same as responses to the original SF-12 items when administered alone. This assumption was tested in two studies, confirming the congruence in mean SF-12 PCS and MCS when the SF-12 was administered on its own compared with when the SF-12 items were extracted from the SF-36 [6, 29, 30].

When calculating the SF-36 PCS and MCS scores, the eight SF-36 domains were factor-analyzed using PCA to obtain the domain weights. Because we assumed the factors to be correlated, we applied oblique rotation, as is recommended in PCA [31]. After oblique rotation, factors indeed showed moderate to high correlations, confirming 
our choice for oblique rotation. By using this rotation method, our domain weights differed from the gold-standard US weights, which used PCA orthogonal rotation [24]. Other studies have also used orthogonal rotation since this procedure is recommended by the developers [21, 23, $24,32]$. When we forced an orthogonal rotation, our weights resembled US weights closely. Importantly, a recent study including over 40,000 subjects from two largescale UK population samples reported that oblique models gave the best fit to the data, and indicated a considerable correlation between PCS and MCS (between .60 and .76 depending on the adhered model) [33]. These findings warrant further detailed examination of the relation between PCS and MCS scores in previous and future studies, as well as examination of the implications for the calculation of the summary scores, and reported results.

Post-MI patients had statistically significant and clinically relevant poorer scores compared with the general Dutch population on MCS and PCS of the SF-12, and after controlling for all covariates, patients still differed significantly from the norm population on PCS scores in oblique but not in orthogonally rotated data. When controlling for covariates, MI did not significantly affect PCS-12 anymore in orthogonally rotated data, although fewer covariates significantly affected PCS (and MCS) scores.

Depression and anxiety were found to significantly explain group differences in PCS-12 scores between the general population and the post-MI population, using the oblique rotation method. This finding may be explained by the inclusion of somatic symptoms such as fatigue and sleeping problems in the definition of depression. In fact, depression in post-MI patients is confounded by cardiac health (i.e., pump function, cardiac history) [34, 35]. Therefore, it was to be expected that depression (and anxiety, a frequent comorbid condition to depression) would act as a significant covariate of the physical summary score.

To our knowledge, only one other study compared health status of MI patients, measured with the SF-12, with normative data [36]. That study also concluded that MCS and PCS scores were significantly lower in patients than in normative controls, although they did not control for possible covariates, nor did they stratify by age.

One possible explanation for these observed differences between the data obtained with the two rotation methods would be differences in SF-12 regression weights. When examining these weights (Table 2), we may observe several differences. The vitality item and the social functioning item have a stronger impact on PCS in the oblique rotation data, compared with their impact on the orthogonal rotation data. In addition, mental health items and the emotional role item load stronger on the PCS in case of orthogonal rotation, compared with the PCS obtained from oblique rotation. When comparing the MCS scores, it is noteworthy that the bodily pain item as well as the physical functioning items load stronger on the orthogonal derived MCS. Concluding, it is clear that, in the orthogonal rotation data, the PCS score is more strongly affected by the mental health questions, compared with the oblique rotation data, while the MCS score is more strongly affected by physical health items, compared with the oblique rotation data. This would mean that, at item level, forcing the summary scores to be uncorrelated (i.e., orthogonal rotation) has the unwanted effect of crossloading of physical and mental items on both the summary scores. This effect has been described previously [25], and now is confirmed in a population of different nationality in the current study.

Our findings demonstrated less pronounced age differences in health status scores with increasing age compared with previous studies $[23,37,38]$. The plateau in healthrelated quality of life may be characteristic of the Dutch population. The difference, for example, compared with US norm scores (which show a steadily decline in PCS and a stable or slightly increasing MCS as people grow older [15], especially regarding PCS), may be explained by differences in health care systems, and social security, but also by cultural differences in health appraisal. Health care in The Netherlands is available for everyone, at an acceptable cost. In addition, social security acts provide a guaranteed income for those who cannot support themselves. Therefore, becoming ill may have a smaller effect on physical health-related quality of life in The Netherlands as compared with, for example, in the USA or other European countries.

The observation in our study that health status was not related to educational level may at first seem odd, since it is known from previous studies that low socioeconomic status (SES) may negatively affect health care utilization in case patients do not have health care insurance. However, in The Netherlands everyone has equal access to care, despite SES, which can explain why a lower educational level was not significantly related to PCS and MCS scores.

Some limitations of the current study should be noted. Overall sample size of post-MI patients $(n=459)$ was rather small in comparison with the Dutch norm population $(n=2,301)$. Furthermore, for post-MI patients our own new Dutch weights were used, which may hamper direct comparison with other studies using different weights. However, as we presented domain weights, regression weights, and norm scores for both rotation methods, our results will be comparable with future studies that use either method. Furthermore, participants in our study were between 30 and 79 years of age, which limits possible comparability with studies outside this age range. 
Despite these limitations, the current study is the first to present Dutch population norms for the SF-12, stratified by age and sex, which can be useful to interpret PCS and MCS scores from other Dutch studies using this instrument. Moreover, this study paves the way for reflection on the potential models relating PCS with MCS, and influencing PCS and MCS calculation. Finally, we showed that Dutch post-MI patients differ significantly from the normative population on PCS scores, even when controlling for disease characteristics, risk factors, and comorbid diseases. In conclusion, this study presents evidence to support the use of the SF-12 as a shorter alternative to the SF-36 in the assessment of health status in Dutch studies, particularly when overall physical and mental health are the main outcomes of interest.

Acknowledgements We would like to thank Dr. Elisabeth J. Martens for providing us with the data on post-MI patients. We wish to thank Dr. Frans Pouwer for his advice on some statistical procedures. Furthermore, we thank all participants for their participation in this study.

Open Access This article is distributed under the terms of the Creative Commons Attribution Noncommercial License which permits any noncommercial use, distribution, and reproduction in any medium, provided the original author(s) and source are credited.

\section{References}

1. Rumsfeld, J. S. (2002). Health status and clinical practice: When will they meet? Circulation, 106(1), 5-7. doi:10.1161/01.CIR. 0000020805.31531.48

2. Spertus, J. (2001). Selecting end points in clinical trials: What evidence do we really need to evaluate a new treatment? American Heart Journal, 142(5), 745-747. doi:10.1067/mhj.2001.11 9135.

3. Spertus, J. A., Radford, M. J., Every, N. R., Ellerbeck, E. F., Peterson, E. D., \& Krumholz, H. M. (2003). Challenges and opportunities in quantifying the quality of care for acute myocardial infarction: Summary from the acute myocardial infarction working group of the American heart association/American college of cardiology first scientific forum on quality of care and outcomes research in cardiovascular disease and stroke. Journal of the American College of Cardiology, 41(9), 1653-1663. doi: 10.1016/S0735-1097(03)00415-7.

4. Ware, J. E., Jr., \& Sherbourne, C. D. (1992). The mos 36-item short-form health survey (sf-36). I. Conceptual framework and item selection. Medical Care, 30(6), 473-483. doi:10.1097/0000 5650-199206000-00002.

5. Ware, J., Jr., Kosinski, M., \& Keller, S. D. (1996). A 12-item short-form health survey: Construction of scales and preliminary tests of reliability and validity. Medical Care, 34(3), 220-233. doi:10.1097/00005650-199603000-00003.

6. Ware, J. E., Kosinski, M., \& Keller, S. D. (1995). How to score the sf-12 physical and mental health summary scales. Boston: The Health Institute, New England Medical Center.

7. Stewart, A., Hays, R., \& Ware, J. (Eds.). (1992). Methods of validating the mos health measures. London: Duke University Press.
8. Aaronson, N. K., Muller, M., Cohen, P. D., Essink-Bot, M. L., Fekkes, M., Sanderman, R., et al. (1998). Translation, validation, and norming of the Dutch language version of the sf-36 health survey in community and chronic disease populations. Journal of Clinical Epidemiology, 51(11), 1055-1068. doi:10.1016/S08954356(98)00097-3.

9. Williams, J. W., Jr., Pignone, M., Ramirez, G., \& Perez Stellato, C. (2002). Identifying depression in primary care: A literature synthesis of case-finding instruments. General Hospital Psychiatry, 24(4), 225-237. doi:10.1016/S0163-8343(02)00195-0.

10. Composite international diagnostic interview (cidi). (1990). Geneva: World Health Organisation.

11. Smeets, R., \& Dingemans, P. (1993). Composite international diagnostic interview (cidi), version 1.1. Amsterdam, The Netherlands: University of Amsterdam.

12. APA. (1994). Diagnostic and statistical manual of mental disorders (4th ed.). Washington, DC: American Psychiatric Association.

13. Wittchen, H. U. (1994). Reliability and validity studies of the who-composite international diagnostic interview (cidi): A critical review. Journal of Psychiatric Research, 28(1), 57-84. doi:10.1016/0022-3956(94)90036-1.

14. Wittchen, H. U., Robins, L. N., Cottler, L. B., Sartorius, N., Burke, J. D., \& Regier, D. (1991). Cross-cultural feasibility, reliability and sources of variance of the composite international diagnostic interview (cidi). The multicentre who/adamha field trials. The British Journal of Psychiatry, 159, 645-653, 658. doi: 10.1192/bjp.159.5.645.

15. Gandek, B., Ware, J. E., Aaronson, N. K., Apolone, G., Bjorner, J. B., Brazier, J. E., et al. (1998). Cross-validation of item selection and scoring for the sf- 12 health survey in nine countries: Results from the iqola project. International quality of life assessment. Journal of Clinical Epidemiology, 51(11), 1171-1178. doi:10.1016/S0895-4356(98)00109-7.

16. McHorney, C. A., Ware, J. E., Jr., Lu, J. F., \& Sherbourne, C. D. (1994). The mos 36-item short-form health survey (sf-36): III. Tests of data quality, scaling assumptions, and reliability across diverse patient groups. Medical Care, 32(1), 40-66. doi:10.1097/ 00005650-199401000-00004.

17. Ware, J. E., Jr. (1993). Sf-36 health survey: Manual and interpretation guide. Boston: The Health Institute, New England Medical Centre.

18. Ware, J., Kosinski, M., \& Keller, S. D. (1994). Sf-36 ${ }^{\circledR}$ physical and mental health summary scales: A user's manual. Boston, MA: The Health Institute.

19. Lam, C. L., Tse, E. Y., \& Gandek, B. (2005). Is the standard sf-12 health survey valid and equivalent for a Chinese population? Quality of Life Research, 14(2), 539-547. doi:10.1007/s11136004-0704-3.

20. Ware, J. E., Jr., Gandek, B., Kosinski, M., Aaronson, N. K., Apolone, G., Brazier, J., et al. (1998). The equivalence of sf-36 summary health scores estimated using standard and countryspecific algorithms in 10 countries: Results from the iqola project International quality of life assessment. Journal of Clinical Epidemiology, 51(11), 1167-1170. doi:10.1016/S0895-4356(98) 00108-5.

21. Jenkinson, C., \& Layte, R. (1997). Development and testing of the uk sf-12 (short form health survey). Journal of Health Services Research and Policy, 2(1), 14-18.

22. Jenkinson, C., Layte, R., Jenkinson, D., Lawrence, K., Petersen, S., Paice, C., et al. (1997). A shorter form health survey: Can the sf-12 replicate results from the sf-36 in longitudinal studies? Journal of Public Health Medicine, 19(2), 179-186.

23. Kontodimopoulos, N., Pappa, E., Niakas, D., \& Tountas, Y. (2007). Validity of sf-12 summary scores in a Greek general population. Health and Quality of Life Outcomes, 5, 55. doi: $10.1186 / 1477-7525-5-55$. 
24. Jenkinson, C. (1999). Comparison of uk and us methods for weighting and scoring the sf-36 summary measures. Journal of Public Health Medicine, 21(4), 372-376. doi:10.1093/pubmed/ 21.4.372.

25. Farivar, S. S., Cunningham, W. E., \& Hays, R. D. (2007). Correlated physical and mental health summary scores for the sf-36 and sf-12 health survey, v.I. Health and Quality of Life Outcomes, 5, 54. doi:10.1186/1477-7525-5-54.

26. Cohen, J. (1988). Statistical power analysis for the behavioral sciences. Hillsdale, NJ: Lawrence Erlbaum Associates.

27. Cohen, J. (1992). A power primer. Psychological Bulletin, 112, 155-159. doi:10.1037/0033-2909.112.1.155.

28. Spindler, H., Kruuse, C., Denollet, J., \& Pedersen, S. S. (2008). Positive and negative effect correlate differently with distress and health related quality of life in Danish cardiac patients: Crosscultural validation of the global mood scale. Psychosomatic Medicine (revised version submitted).

29. Muller-Nordhorn, J., Roll, S., \& Willich, S. N. (2004). Comparison of the short form (sf)-12 health status instrument with the sf-36 in patients with coronary heart disease. Heart (British Cardiac Society), 90(5), 523-527. doi:10.1136/hrt.2003.013995.

30. Schofield, M. J., \& Mishra, G. (1998). Validity of the sf-12 compared with the sf-36 health survey in pilot studies of the Australian longitudinal study on women's health. Journal of Health Psychology, 3(2), 259-271. doi:10.1177/135910539800 300209.

31. Stevens, J. (1996). Applied multivariate statistics for the social sciences (Vol. 3). Mahwah, NJ: Lawrence Erlbaum Associates, Inc.
32. Jenkinson, C. (1998). The sf-36 physical and mental health summary measures: An example of how to interpret scores. Journal of Health Services Research and Policy, 3(2), 92-96.

33. Hann, M., \& Reeves, D. (2008). The sf-36 scales are not accurately summarised by independent physical and mental component scores. Quality of Life Research, 17(3), 413-423. doi: 10.1007/s11136-008-9310-0.

34. de Jonge, P., Denollet, J., van Melle, J. P., Kuyper, A., Honig, A., Schene, A. H., et al. (2007). Associations of type-d personality and depression with somatic health in myocardial infarction patients. Journal of Psychosomatic Research, 63(5), 477-482. doi:10.1016/j.jpsychores.2007.06.002.

35. Martens, E. J., Smith, O. R., \& Denollet, J. (2007). Psychological symptom clusters, psychiatric comorbidity and poor self-reported health status following myocardial infarction. Annals of Behavioral Medicine, 34(1), 87-94. doi:10.1007/BF02879924.

36. Crilley, J. G., \& Farrer, M. (2001). Impact of first myocardial infarction on self-perceived health status. Quarterly Journal of Medicine, 94(1), 13-18. doi:10.1093/qjmed/94.1.13.

37. Augustovski, F. A., Lewin, G., Elorrio, E. G., \& Rubinstein, A. (2008). The argentine-spanish sf-36 health survey was successfully validated for local outcome research. Journal of Clinical Epidemiology, 61(12), 1279-1284, e1276.

38. Hopman, W. M., Towheed, T., Anastassiades, T., Tenenhouse, A., Poliquin, S., Berger, C., et al. (2000). Canadian normative data for the sf-36 health survey. Canadian multicentre osteoporosis study research group. Canadian Medical Association Journal, 163(3), 265-271. 